\title{
Mehr Bindungsstellen für Antidepressiva
}

Die Anzahl von Serotonintranportern im Gehirn wird durch Testosteron erhöht.

Männer leiden in höherem Alter, wenn die Ausschüttung des Geschlechtshormons Testosteron abnimmt, häufiger an Depressionen und einige Studien konnten bereits einen positiven Effekt einer Testosterongabe auf die Stimmung der Betroffenen nachweisen. Nun konnten Forscher der MedUni Wien erstmals zeigen, dass Testosteron die Anzahl von Serotonintransportern im menschlichen Gehirn erhöht. Diese Proteine regeln die Konzentration von Serotonin und sind auch der Zielort von Antidepressiva.

Als Modell für die Untersuchung der Testosteronwirkung wählten die Forscher der MedUni Wien die Hormontherapie von Transsexuellen. Erstautor Mag. Dr. Georg S. Kranz: „Transsexuelle sind Menschen, die das Gefühl haben, im falschen Körper zu leben und deshalb eine hochdosierte gegengeschlechtliche Hormontherapie wünschen, um ihr Erscheinungsbild an das jeweils andere Geschlecht anzupassen. Genetische Frauen erhalten Testosteron, genetische Männer Östradiol sowie Medikamente zur Unterdrückung von Testosteron."

Mittels PET konnten die Wissenschaftler nachweisen, dass die Serotonintransporter im Gehirn bereits nach vierwöchiger Hormontherapie mit Testosteron signifikant erhöht sind und bei fortlaufender Therapie weiter ansteigen. Weiters konnte ein enger Zusammenhang zwischen Testosteron im Blut und der Serotonintransporterdichte nachgewiesen werden.

Die Studie hat gezeigt, dass Testosteron die möglichen Bindungsstellen für häufig verschriebene Antidepressiva wie SSRIs im Gehirn erhöht und er-

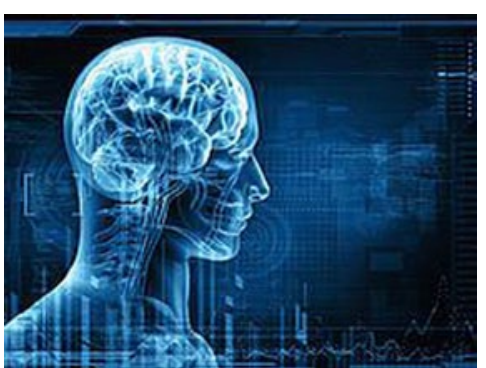

laubt damit wesentliche Einblicke in die Wirkung von Geschlechtshormonen auf das menschliche Gehirn und Geschlechtsunterschiede bei psychiatrischen Erkrankungen.

psychopraxis.neuropraxis 2015 · 18:63 DOI 10.1007/s00739-015-0249-y

Online publiziert: 21. März 2015

○ Springer-Verlag Wien 2015
Quelle: MedUni Wien

Kranz GS et al.: High-Dose

Testosterone Treatment

Increases Serotonin Transporter Binding in Transgender People. Biol Psychiatry. 2014 Sep 23. pii: S00063223(14)00709-4. doi: 10.1016/j.biopsych.2014.09.010. [Epub ahead of print] 